\title{
Artikel
}

Peter J. Schneemann*

\section{Emanzipierte Rezeption. Kopie und Reproduktion als produktive Kulturtechnik der Mittelbarkeit}


Abstract: Die künstlerische Kopie bietet nicht erst in der zeitgenössischen Kunst ein Verfahren der Aneignung und Umdeutung. In der Fokussierung auf aktuelle künstlerische Strategien verfolgt der Aufsatz die These, dass die Kopie nicht nur Produktionsmodi verhandelt, sondern auch spezifische Paradigmen einer emanzipierten Rezeption. An den Schnittstellen von Reproduktion und Kopie, Zitat und Aneignung, Reenactment und Preenactment finden sich neue Wahrnehmungshaltungen und -handlungen ausdifferenziert. Das Potenzial dieser Ästhetik der Mittelbarkeit führt zu Fragen der Emanzipation und Differenz.

Keywords: Reproduktion, Rezeptionsästhetik, Museum, zeitgenössische Kunst, Mittelbarkeit.

*Prof. Dr. Peter J. Schneemann, Institut für Kunstgeschichte, Abteilung für Kunstgeschichte der Moderne und der Gegenwart, Mittelstrasse 43, CH-3012 Bern, email: peter.schneemann@ikg.unibe.ch

\section{Fragmentierte Referenzen}

Das Publikum betrachtet große skulpturale Fragmente, die auf Paletten liegen oder durch Hilfskonstruktionen gestützt werden. Monumentale Faltenwürfe, geformt aus dünnen Kupferplatten, finden sich neben Ausformungen einer Krone oder Fingern einer Hand (Abb. 1). Der Ausstellungsraum erscheint als Lager, das einen zerlegten Körper beherbergt. Die Stücke aktivieren Erinnerungen an Postkartenabbildungen und die popkulturelle Präsenz eines der bekanntesten monumentalen Wahrzeichen. Selbst das Erlebnis einer Hafenrundfahrt in New York belegt, wie stark der Prozess des Wiedererkennens an die unzähligen Abbildungen und kleinformatigen Repliken gebunden ist, durch die sich die Freiheitsstatue aus dem 19. Jahrhundert ins kollektive Bildgedächtnis eingeschrieben hat.

Das Projekt We The People (Detail) (2011-16) des Künstlers Danh Võ veranschaulicht spezifische Potenziale der Kopie, denen sich mein Beitrag im Folgenden nähern wird. Das über mehrere Jahre entwickelte Projekt einer 1:1 Replik des 1886 eingeweihten, ikonischen Denkmals von FrédéricAuguste Bartholdi mündete in eine Serie von Ausstellungen, die je eine Auswahl aus den über 300 Fragmenten an unterschiedlichsten geografischen
Orten zeigte. ${ }^{1}$ Die in Shanghai im Auftrag des Künstlers angefertigten Stücke verteilen sich auf über fünfzehn Länder und zielten zu keinem Zeitpunkt auf Vervollständigung zu einer Figur.

Den Körperfragmenten fehlt die haltende Konstruktion, die für das begehbare Monument entscheidend war - die Kopie besteht nur aus der reinen Oberfläche. Das Erleben der Kopie-Fragmente, die in ihrer Materialität des glänzenden Kupfers aus der Nähe, auf dem Boden liegend, sich dem Publikum darboten, steht im Kontrast zur Referenz des Originals in New York. Die Statue of Liberty gehört zu den Denkmälern, die aus der Ferne rezipiert wurden und als Turm dienten, in dessen Innerem die Besucher_innen emporsteigen, um aus der Figur heraus auf die Welt zu schauen. ${ }^{2}$ Die Anagrammatik Danh Võs befragt nicht nur den Status des Originals, sondern auch die Idee seiner Ortsspezifik. ${ }^{3}$

1 Danh Võ, We The People, ausgewählte Ausstellungsorte: Kassel Fridericianum 2011, The Art Institute of Chicago 2012, National Gallery of Denmark 2012, Peer London 2013, Faurschou Foundation Beijing 2014, Brooklyn Bridge Park \& City Hall Park NYC 2014, Museum Ludwig Köln 2015, Solomon R. Guggenheim Museum 2018; vgl. Dziewior 2016. 2 Erben 2005.

3 Es mag daran erinnert werden, dass die ersten Präsentationen zur Statue of Liberty ebenfalls in der Form von 


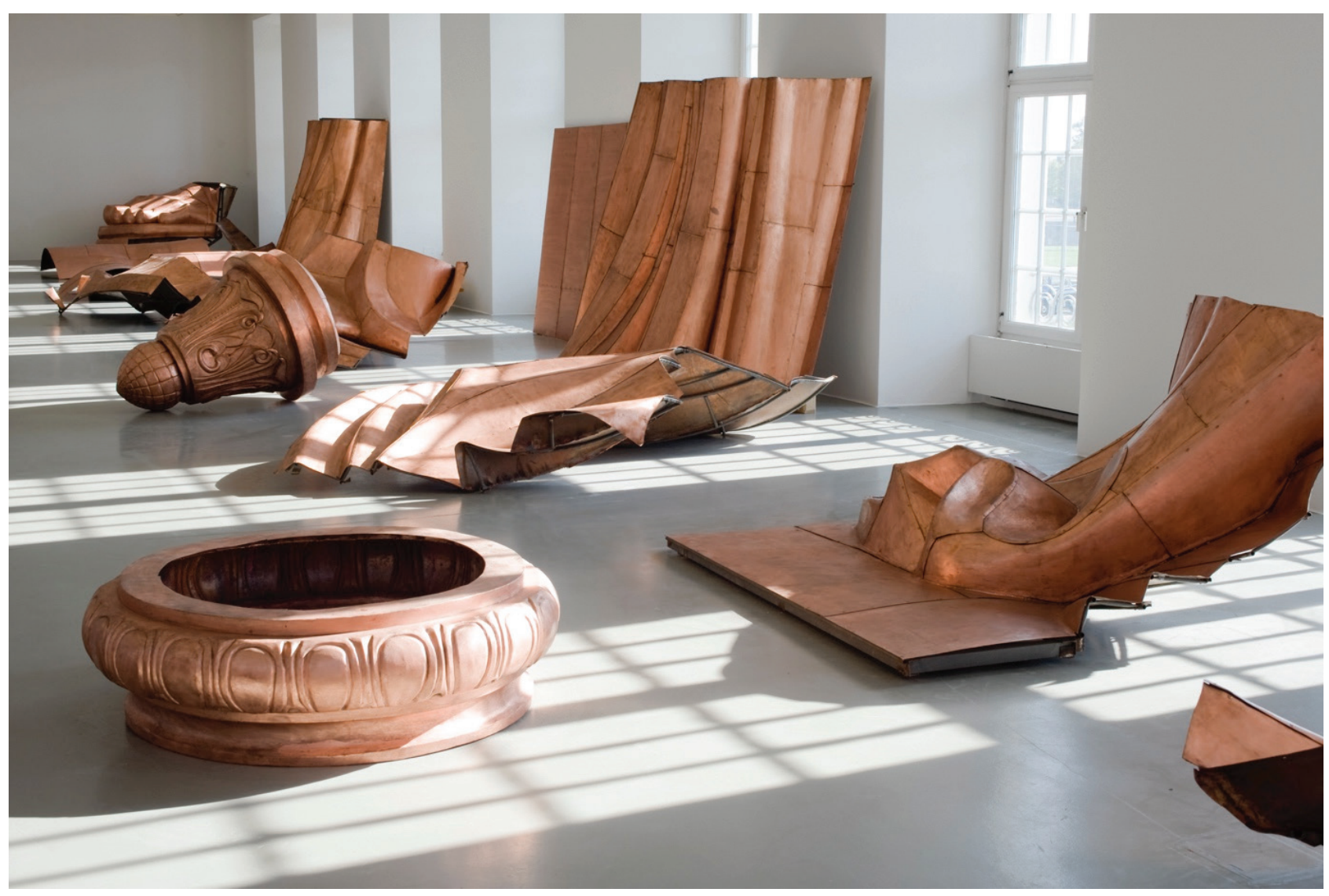

Abb. 1. Danh Võ, We The People (Detail), 2011-2016, Ausstellungsansicht, JULY, IV, MDCCLXXVI, Kunsthalle Fridericianum, Kassel, 2011. Foto: Nils Klinger

Courtesy of Kunsthalle Fridericianum, Kassel and Galerie Chantal Crousel, Paris.

In der mehrfachen Dekontextualisierung aus dem historisch-geografischen Kontext und der Rekontextualisierung in einem jeweilig anderen Rezeptionsprozess finden bedeutende semantische Verschiebungen statt. Die Kopien werden in dem Land erstellt, das die Kompetenz und Ökonomie der Nachbildung bereits im 16. Jahrhundert entwickelte. ${ }^{4}$ Die Fragmente aus China werden anschließend auch zurück in den Westen transportiert und geografisch verteilt. Es ist das große Thema der Migration, das Danh Võ immer wieder via Objekte und autobiografische Narrationen verhandelt. We The People nutzt als künstlerische Strategie den Prozess des Nachformens.

Fragmenten geschah: So wurde die Fackel auf der Centennial Exhibition 1876 in Philadelphia gezeigt und der Kopf auf der Weltausstellung 1878 in Paris. Zur Finanzierung wurden auch Modelle verkauft. Auch die Tatsache, dass die in Paris vorgefertigten Teile in New York zusammengefügt wurden, gehört zum Subtext von Danh Võs Projekt.

4 Vgl. Henningsen / Hofmann 2012.
Dabei entfaltet sich auch eine Reflexion über die gesellschaftliche Funktion der Kopie als Reproduktion, die neue Vermittlungskontexte und Zielsetzungen erschließt. Der Fokus auf die Momente der Rezeption soll im Folgenden den hohen produktionsästhetischen Stellenwert des Kopierens in der Geschichte der Kunstausbildung ergänzen und den komplexen Prozessen von Zitat und Aneignung als künstlerische Auseinandersetzung die Potenziale für das Publikum als eine neue Perspektive zur Seite stellen. Das gegenwärtige künstlerische Interesse an der Leistung von Replik und Reenactment zielt auf emanzipatorische Prozesse der Verhandlung von Kultur. ${ }^{5}$

Die Fragestellung dieses Beitrags richtet sich auf die Rezeption von Kopien und Reproduktionen als eine Wahrnehmung zweiter Ordnung. Die Mittelbarkeit in der Betrachtung von und Umgang mit der Realität des Kunstwerks erweist

5 Von Gehlen 2012, S. 323-333. 
sich als eine spezifische Form der Reflexion mit den Mitteln von Wiederholung und Differenz. Es wird die These verfolgt, dass der Umgang mit dem Status der Kopie eine Kulturtechnik des Sekundären anzeigt. ${ }^{6}$ Sie betrifft den zeitgenössischen Umgang mit und Zugang zur Realität. ${ }^{7}$ Damit befrage ich die wichtige These Hans Ulrich Gumbrechts, der so vehement die Würdigung einer Präsenz fordert, die jenseits der Interpretation anzusiedeln sei. ${ }^{8}$ Diese Konstellation von Präsenz und Wirkung, Reproduktion und Reflexion erscheint nicht zuletzt deshalb so virulent, weil bereits André Malraux im Kontext seiner Überlegungen zu einem Musée Imaginaire als Konsequenzen der Reproduktion eine befreite ,Intellektualisierung' im Umgang mit der Kunst prophezeite. ${ }^{9}$ In welcher Weise entspricht also die Kopie einem aktuellen Modus der Kunstrezeption, der jenseits einer kulturpessimistischen Perspektive in seiner Leistungsfähigkeit zur Veränderung zu beschreiben ist?

Meine Überlegungen finden ihren Ursprung in einer Tagung, die 2013 im Otsuka Museum of Art in Naruto, Japan, stattfand. ${ }^{10}$ Dieses Museum versammelt den Kanon der westlichen Kunstgeschichte von Giotto bis Andy Warhol ausschließlich in Form von Reproduktionen (Abb. 2). Angefertigt unter der Mitwirkung des kunsthistorischen Institutes in Tokyo, sind diese auf Keramikplatten fixiert. Die Tagung, deren Hauptsektionen im Nachbau der Sixtinischen Kapelle stattfanden und deren Präsentationen auf die Kopie des Jüngsten Gerichts projiziert wurden, trug den Titel Between East and West. Reproductions in Art. Die Reproduktion erweist sich als das Medium, das eine entscheidende Rolle im interkulturellen Verständnis spielt. Wie kann die Betrachtung einer Nachbildung zum kritischen Bewusstsein einer bedingten Verfügbarkeit führen? Inwieweit ist der Umgang mit dem Modus des ,Vermittelten' an eine aktivierte und emanzipierte Rezeption zu knüpfen?

\section{Übungen der Mittelbarkeit}

Die künstlerischen Affirmationen ebenso wie Dekonstruktionen des Dualismus von Original und Kopie sind zahllos und ausgiebig studiert. Dabei spielen Begrifflichkeiten und Wertzuschreibungen eine nicht zu vernachlässigende Rolle. Das Vokabular für die Bezeichnung der Beziehung zwischen einem Bild und seinem Referenzbild verrät die konzeptuellen Implikationen. Kuratorische Bearbeitungen des Themas operieren mit Typologien und Glossaren: ,Auftragskopie', ,Appropriation Art', ,Hommage', ,Parodie', ,Wiederholung als Ritual'.11 Jede der Kategorien können Beispielen zugeordnet werden und Künstler wie Marcel Duchamp müssen gleich für verschiedene Aspekte der Kopie als Referenz dienen. Es werden Unterscheidungen vorgenommen nach Funktion, nach Motivation und Status. Das Nachbilden wird etwa als Übung für die jungen Künstler_innen beschrieben - die Annäherung an die alten Meister als Einschreibung in eine stringente stilistische Entwicklung. Die École nationale supérieure des beaux-art in Paris operierte zu diesem Zwecke mit einem eigenen Museum der Kopien. ${ }^{12}$

Eine völlig andere, moderne Variante bezieht sich dagegen auf die Kopie als eine Strategie der Aneignung, der subversiven Dekonstruktion. Die Vervielfältigung zielt dabei als kritische Relektüre auf eine Offenlegung verdeckter ideologischer Implikationen. So ist es etwa das Konzept der Autorschaft, welches die Diskurse um die Appropriation adressieren. Eklektizismus, Wiederholung, Kopie erscheinen als Dekonstruktionen des Geniekultes und des Wertesystems von Kreativität. ${ }^{13}$ Die Doktrin der Inventio und des Unikats weicht Techniken des Samplings. ${ }^{14}$

Auf der Ebene der Konzepte der Moderne bleibt also schlicht zu konstatieren, dass die Vorstellung von einem ersten Bild als dem Ursprung vom zweiten Bild längst aufgegeben und vielmehr der Fokus auf Prozesse der Umdeutung und permanenten Rückwirkung gelenkt werden muss. ${ }^{15}$ Die ideologischen Implikationen der Begrifflichkeiten

11 Vgl. Mensger 2012; Schwartz 2000.

12 Vgl. Boime 1964.

13 Vgl. Römer 2001; Gelshorn 2012; Han 2011.

$14 \mathrm{Vgl}$. Weibel 1999.

15 Vgl. Blunck 2011.
7 Es ist die wichtige Ausstellung More Real? Art in the Age of Truthiness zu erwähnen. Vgl. dazu Armstrong 2012.

8 Vgl. Gumbrecht 2004a; Gumbrecht 2004b; Mersch 2006. 9 Vgl. Malraux 1949; Grasskamp 2014. 


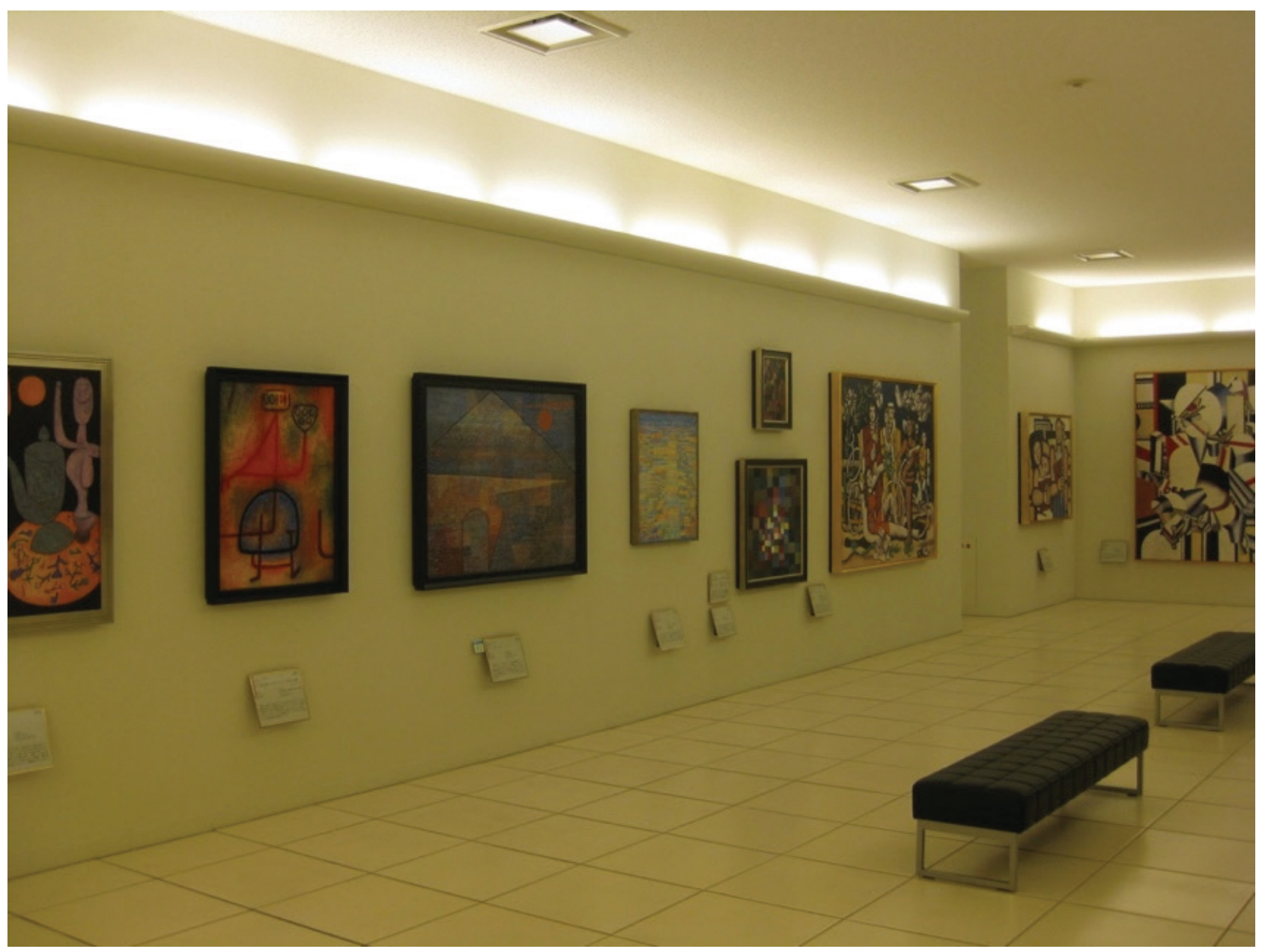

Abb. 2. Ausstellungsansicht Otsuka Museum of Art, Naruto, Japan, 2013.

Foto: Peter J. Schneemann

verweisen jedoch nicht nur auf die Ansätze einer kritischen Revision, sondern zeigen eine weitere Ebene an, die heute ungeahnte Aktualität besitzt. Die Kopie steht für eine Kultur der Mittelbarkeit, eines Referenzsystems, das sich vom Status des Originals emanzipiert hat und sich seiner Nachgeordnetheit entzieht. Wir sind mit Kopien konfrontiert, die aus den Mechanismen einer globalen und vielfältigen Öffentlichkeit generiert und innerhalb dieser Regelwerke verbreitet und verhandelt werden. Kopien beziehen sich längst auf Kopien. Dabei besteht ein radikalisierter Zusammenhang zwischen Kopie und Rezeptionsqualität als einem aktiven Prozess, einem gesellschaftlichen Prozess. Man mag einwenden, dass es sich zunächst um einen Allgemeinplatz handle, der die gesamte Kunstgeschichte beträfe. Dennoch ist es kein Zufall, dass die Reproduktionskopie immer wieder im Kontext neuer Technologien, sei es dem Kupferstich oder der Fotografie, disku- tiert und dabei der Begriff der Wirkung als Kriterium befragt wurde, das ideologisch konnotierte Bewertungen von Distribution impliziert. ${ }^{16}$

Dirk von Gehlen publizierte 2011 unter dem Titel Mashup eine Bestandsaufnahme der Kopie in der zeitgenössischen Kultur. ${ }^{17}$ Sein Index zeigt die Präsenz der Kopie als Praxis in allen Feldern der Populärkultur und der Hochkultur - mit Implikationen, die bis in politische und juristische Bereiche reichen. Er erweitert nicht nur nochmals den Eindruck der Unmenge von Differenzierungen und Bezeichnungen von Neologismen, sondern auch den Transfer von Bereichen der Musik in die allgemeine Kunstpraxis. ,Mashup', ,Remix', ,Reprise', ,Sample'.18

16 Vgl. Lambert 1987; Ullrich 2015.

17 Vgl. von Gehlen 2012, S. 7.

18 Von Gehlen 2012. 
Der Kopie haftet dennoch immer noch der Moment des Verlustes, des Ersatzes und Scheiterns an. Viele der Bezeichnungen zeigen neben der Funktion vor allem auch einen Status im Wertesystem an, der geprägt ist von der Idee des minderwertigen ,Ersatzes': ,Replik', ,Imitation', ,Mimikry', ,Fake', ,Zitat', ,Placebo', ,Surrogat'. Die neue Auseinandersetzung mit der Reproduktion demonstriert dagegen das Gewicht einer sich selbst thematisierenden Bedingtheit. Bei Danh Võ ist es die Fragmentierung, geografisch potenziert, die diesen Aspekt der Kopie adressiert. Es erscheint ebenso unmöglich wie auch hinfällig zu sein, die Fragmente wieder zu einer Gesamtfigur zusammenzufügen. Die Wahrnehmung des Kunstwerkes als Kopie verschränkt die Gegenwart des Augenblicks mit Dimensionen der Erinnerung. ${ }^{19}$ Anachrone Qualitäten durchbrechen Vorstellungen einer linearen Logik in der Chronologie der Erfahrung. ${ }^{20}$ Die Reproduktion bietet sich einem ständig wechselnden Kontext an. Emanzipierte Handlungen, Missverständnisse und neue Rahmungen durchbrechen über die Mittelbarkeit des Abbildes Aura und Distanz des unberührbaren Originals.

Der Anspruch der absoluten Gleichzeitigkeit wird ebenso aufgegeben wie die Erwartung einer reinen, das heißt unvermittelten Präsenz. Stattdessen erstarkt der Moment der Mittelbarkeit. An dieser Stelle findet etwa ein Aufleben von Techniken der Anekdote und der Ekphrasis statt. ${ }^{21}$ Gegenstand der Betrachtung und Evaluation ist eine ebenso spektakuläre wie fiktionalisierte Rezeptionsgeschichte, die eine neue Eigentlichkeit und Valenz darstellt. In aller Radikalität kann dies anhand der eindrücklichen Ausstellung Lost Art nachvollzogen werden, mit der sich die Tate Gallery 2013 verlorenen oder genauer, abwesenden Kunstwerken widmete. ${ }^{22}$ Dabei entfaltete sich eine ganze Typologie der Absenz, die vom unrealisierten bis zum zerstörten, vom ephemerem bis zum geraubten Werk reichte. Konsequenterweise handelte es sich dabei um eine virtuelle Ausstellung, die ausschließlich über das Internet rezi-

19 Die Konstruktion einer künstlichen ,Gleichzeitigkeit' feierte der Medienkünstler Christian Marclay mit The Clock als monumentale Collage. Vgl. Leader 2010.

20 Vgl. Kernbauer 2015.

21 Vgl. Schneemann 2019.

22 Vgl. Mundy 2013. piert werden konnte. Den Kuratierenden gelang es, Präsenz und Absenz als produktive Dialektik zu entwickeln. Die Ausstellung zeigte die eigenständige Ästhetik eines komplexen Referenzsystems: Dokument und Narration, Absenz und Spur, Beleg und Imagination.

Die ästhetische Erfahrung wird also in der Dekonstruktion einer reinen Unmittelbarkeit keinesfalls ausgeschlossen, aber sie kann als eine geliehene, eine bereits erprobte und zu erprobende beschrieben werden. Ich möchte diese These mit dem Verweis auf den Trend zu Reinszenierungen von Kunstausstellungen veranschaulichen. Zum einen geht es dabei, wie im Falle von Harald Szeemann, um die Würdigung einer kuratorischen Leistung. Zum anderen findet aber eine komplexe Historisierung und Wiederaufführung der Kunstwahrnehmung selbst statt. Diese stellt sich besonders radikal in der Verortung der Leistung der Kopie dar. Die Reinszenierung der Berner Ausstellung von 1969 in den Räumen der Prada Foundation in Venedig operierte mit Originalen ebenso wie mit Markierungen von Absenz. ${ }^{23}$ Doch Teile des Ausstellungsraumes, der Bodenbelag oder die Heizkörper, erwiesen sich als Kopien des ursprünglichen Ausstellungsraumes, hergestellt vom Künstler Thomas Demand (Abb. 3). Diese Autorschaft ist insofern von Bedeutung, als dass Demand durch die Übersetzung von Pressebildern in dreidimensionale Papierskulpturen bekannt geworden ist, die inm ausschließlich als Vorlage für eine Nachbildung, eine Wiederholung und Rekonstruktion der ursprünglichen Fotografie dienten. Die Säle der Kunsthalle wurden in die Räume des Palazzo eingewoben. Als Vorlage dienten die inzwischen ikonisch gewordenen Aufnahmen aus den 1960er Jahren. Nicht die Zusammenstellung der Kunstwerke stand im Interesse der Besucher_innen, sondern die Legacy der Ausstellung.

Die Reinszenierungswelle in der kuratorischen Praxis fördert eine Kunstbetrachtung, die als Rekonstruktion einer historischen Kunsterfahrung an einem anderen Ort zu beschreiben ist. ${ }^{24}$ Doch es geht um mehr. Die Verschiebung von der reinen Kopie zur inszenierten Uneigentlichkeit

23 Hierzu wurden gestrichelte Umrisse im Ausstellungsraum angebracht.

24 Celant 2013; vgl. auch Celant 2015; Conte 2018; Bismarck 2014. 


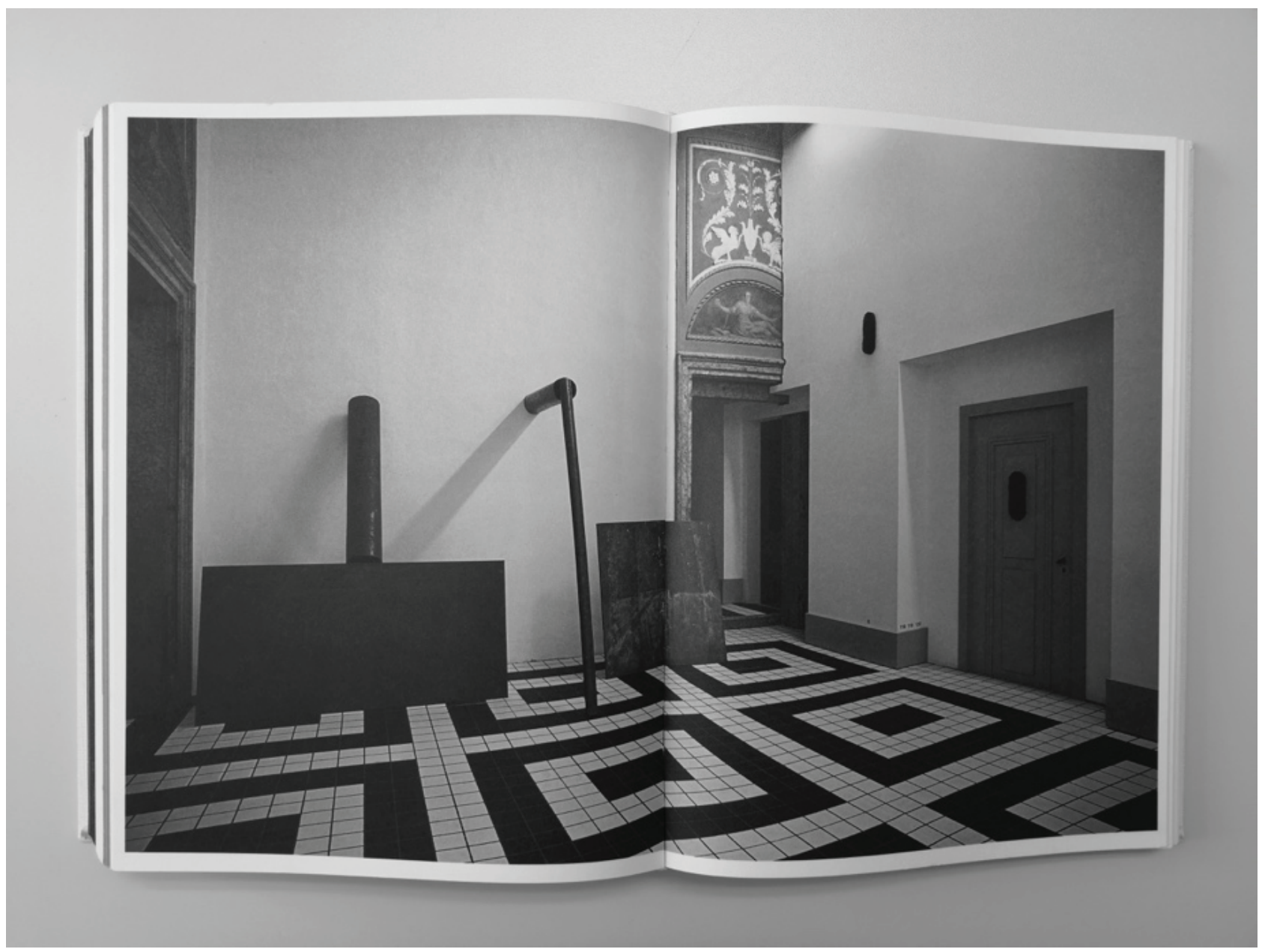

Abb. 3. Ausstellungsansicht When Attitudes Become Form: Bern 1969/Venice 2013. Richard Serra, Shovel Plate Prop, 1969; Close Pin Prop, 1969; Sign Board Prop, 1969 (1987). Richard Artschwager, Blp, 1968.

Abgedruckt in Germano Celant \& Fondazione Prada (Hg.) (2013): When Attitudes Become Form, Bern $1969 /$ Venice 2013. Mailand: Progetto Prada Arte 2013, S. 602-603.

lässt in der Konstellation von Kopie, Reproduktion, Rekonstruktion und Abweichung den emanzipierten Rezipienten als Akteur in den Vordergrund treten. Die so kontrovers diskutierte Wiederholung zeitigte dabei nicht nur in der mehrfachen Potenzierung der Besucherzahlen eine ganz eigene Realität.

\section{Akteurinnen und Akteure der Differenz}

Die Kopie, in Parallelität zur Übersetzung, erweist sich als Kulturtechnik der Vergegenwärtigung, die Variation und Differenz ermöglicht. Den Schritt, das Historische auf die Gegenwart zu beziehen, beschreibt die kunstgeschichtliche Hermeneutik als zentrale Leistung der Deutung. ${ }^{25}$ Interessant ist nun, die performative Qualität in den Benennungen der Kopie wiederzufinden. An die Stelle der Kategorisierung des Status eines Objektes tritt die Bezeichnung von Perspektiven, Zielsetzungen und Strategien. Die Kopie führt weniger zu Objektklassen und Substantiven, sondern vielmehr zur Anzeige von Tätigkeiten. Es erweist sich als fruchtbar, nicht auf Festschreibungen zu fokussieren, sondern vielmehr Aktivierungen zu benennen: Zitieren durch Aneignen, Wiederholen durch Nachahmen, Deuten durch Einschreibung.

Die Reproduktion wird zu einer Referenz, als Wiederholung befragt sie Kategorien der Wertschätzung, spiegelt Modi des Verstehens und

25 Vgl. Bätschmann 1984. Vgl. auch Roesler-Friedenthal / Nathan 2003. 
Missverstehens. Ob Sampling oder Wiederholung, Vervielfältigung oder Übersetzung, wir sind mit Qualitäten des Performativen konfrontiert. Es geht um nichts weniger als um das Angebot und die Praxis polyperspektivischer Lektüren und ihrer gesellschaftlichen Realität.

Es ist in diesem Zusammenhang nicht zufällig, dass sich, nicht zuletzt durch künstlerisches Interesse an dieser Gattung, die Modi der Reproduktion erweitert haben. Wurde früher jede Form von Rahmung und Kontext beim Reproduzieren vermieden, steigt das Interesse, eine Kontextualisierung zu leisten und Rezeptionsprozesse im sogenannten ,Installation Shot', das heißt im Moment eines spezifischen Zeigens, als Aufführung, zu dokumentieren. ${ }^{26}$ Die Darstellung der räumlichen Situation ohne Rezipienten wechselt zu inszenierten Interaktionen mit Statisten und Statistinnen (Abb. 4a, $4 b, 4 c){ }^{27}$ Es entstehen neue Kunstwerke, in die Betrachtungsmodi eingeschrieben sind. Der Bogen der Argumentation führt zur These, dass durch diese Einbindung der Rezeptionsmodi eine aktive, emanzipierte Rezeptionsweise gefordert wird, die über ihre eigenen Bedingtheiten reflektiert.

Die Reproduktionen schreiben die historischen und geografischen Lektüren in ihrer Vielfältigkeit und ihren Projektionen in die Diffusion des Werkes ein. Oder anders gesagt, an die Stelle des Interesses an der Reproduktion des isolierten Werkes tritt der Blick auf den Kontext und den Akt des Schauens. In spielerischer Weise hat diese Betrachtung von Betrachtenden die Künstlerin Georgia Kotretsos anhand der Museumsserie von Thomas Struth bearbeitet (Abb. 5). ${ }^{28}$ Wir haben es mit einem Prozess der Einschreibung zu tun, eine Einschreibung, die Bild und Rezeption so miteinander verschränkt, dass eine endlose Folge vom Bild im Bild entsteht, jedes Mal den Kontext der Rezeption einschließend. Als Reproduktion dokumentiert es die Inszenierung der Wertschätzung, das soziale Event als Ritual mit eigenen Bühnenbildern. Und wiederum können wir beobachten, wie dieser bildgewordene Blick weitere Bildproduktionen als ,Wahrnehmungsvorlagen' auslöst.

Diese Prozesse der Mittelbarkeit sind es, die zunehmend die Vermittlungsarbeit von Museen

26 Vgl. auch die wichtigen Arbeiten von Louise Lawler.

27 Vgl. Sheldon 2017.

28 Vgl. Struth / Belting / Grasskamp 1993. Zu Kotretsos vgl. Kihm 2015. beschäftigt. Mit Tweetups wird versucht, den Umgang mit den sozialen Medien in das Museumserlebnis zu integrieren. ${ }^{29}$ Das sich am schnellsten entwickelnde Format der analysierten Einschreibung, der Rekontextualisierung des Originals durch Aneignung, ist ohne Zweifel das Kunst-Selfie. 2014 publizierte das New Yorker Künstlerkollektiv DIS, das der Post-Internet-Kunst zugeordnet wird, ein Buch mit dem Titel \#artselfie. ${ }^{30} \mathrm{Es}$ demonstriert als Sammlungsgut die ganze Typologie der Parallelisierungen und Überblendungen vom Selbstbild mit dem Kunstwerk. Unmittelbar wird nun deutlich, dass wir in den digitalen Multiplikationen, die Betrachter_innen der Kunst nicht länger exklusiv als Rückenfiguren in den sich multiplizierten Bildern finden, wie sie in der Kunstgeschichte kanonisch wurden. Stattdessen richten sie den Blick aus dem Bild als emanzipiertes Gegenüber, eingeschrieben in die Interaktionen mit dem Kunstwerk. ${ }^{31}$ Die neue Bildpolitik greift die alte Praxis der Tableaux Vivants ebenso auf, wie sie spiegelnde Oberflächen dazu verwendet, die Reproduktion des Werkes mit der Identität der Rezipienten und Rezipientinnen zu überblenden.

Bei Tweetups und Selfies setzt gerne ein kulturkritischer Diskurs ein, der das Erlebnis aus ,zweiter Hand' als großen Verlust beschreibt, als Verlust der Präsenz. Es lohnt sich jedoch der Blick auf den Umgang mit der Reproduktion im Rahmen einer globalisierten Kunstproduktion und Kunstrezeption. Die Kopie als Medium einer emanzipierten Rezeption kann in Verbindung gebracht werden mit der Auflösung von sozialen und geografischen Hierarchien, von alten Vorstellungen von Zentrum und Peripherie.

2013 wurde die ambitionierte Ausstellung Nothing to Declare als Ergebnis eines größeren Forschungsprojektes zur globalisierten Kunstwelt präsentiert. ${ }^{32}$ In diesem Rahmen fand etwa das Video von Araya Rasdjarmrearnsook Dow Song Duang seinen Platz, in dem Édouard Manets Le déjeuner sur l'herbe und andere Werke aus der europäischen Kunstgeschichte des 19. Jahrhun-

29 Vgl. Kohle 2018.

30 Vgl. Castets / Coupland / DIS 2014.

$31 \mathrm{Vgl}$. die präzise Verwendung dieser Strategien in der Louvre-Choreographie von Beyoncé und Jay-Z 2018.

32 Zentrum für Kunst und Medientechnologie 2011 und Zentrum für Kunst und Medientechnologie 2013. Araya Rasdjarmrearnsook, Dow Song Duang - The Two Planets Series, 2008, Video, 17 min. 


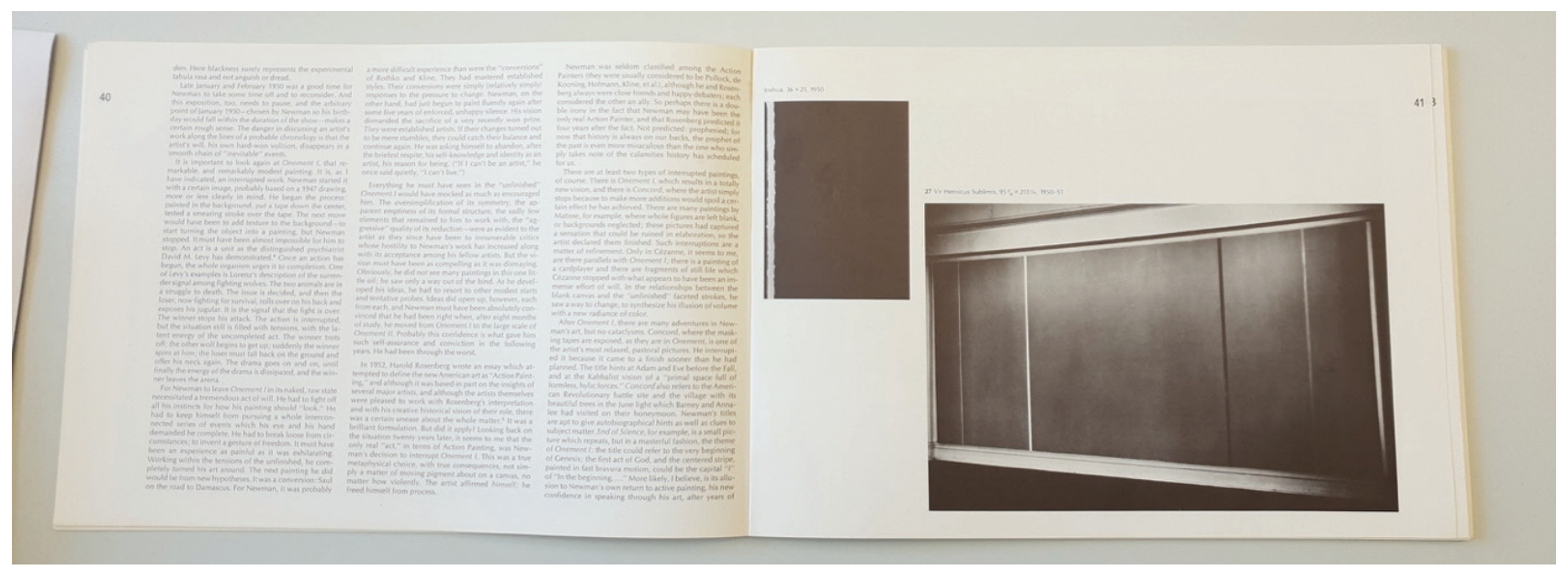

Abb. 4a. Seitenansicht des Ausstellungskataloges Thomas B. Hess und Tate Gallery (Hg.) (1972): Barnett Newman. Ausst.-Kat., London. The Tate Gallery, 28.06-06.08.1972, London: The Tate Gallery Publications Department, S. 40-41.

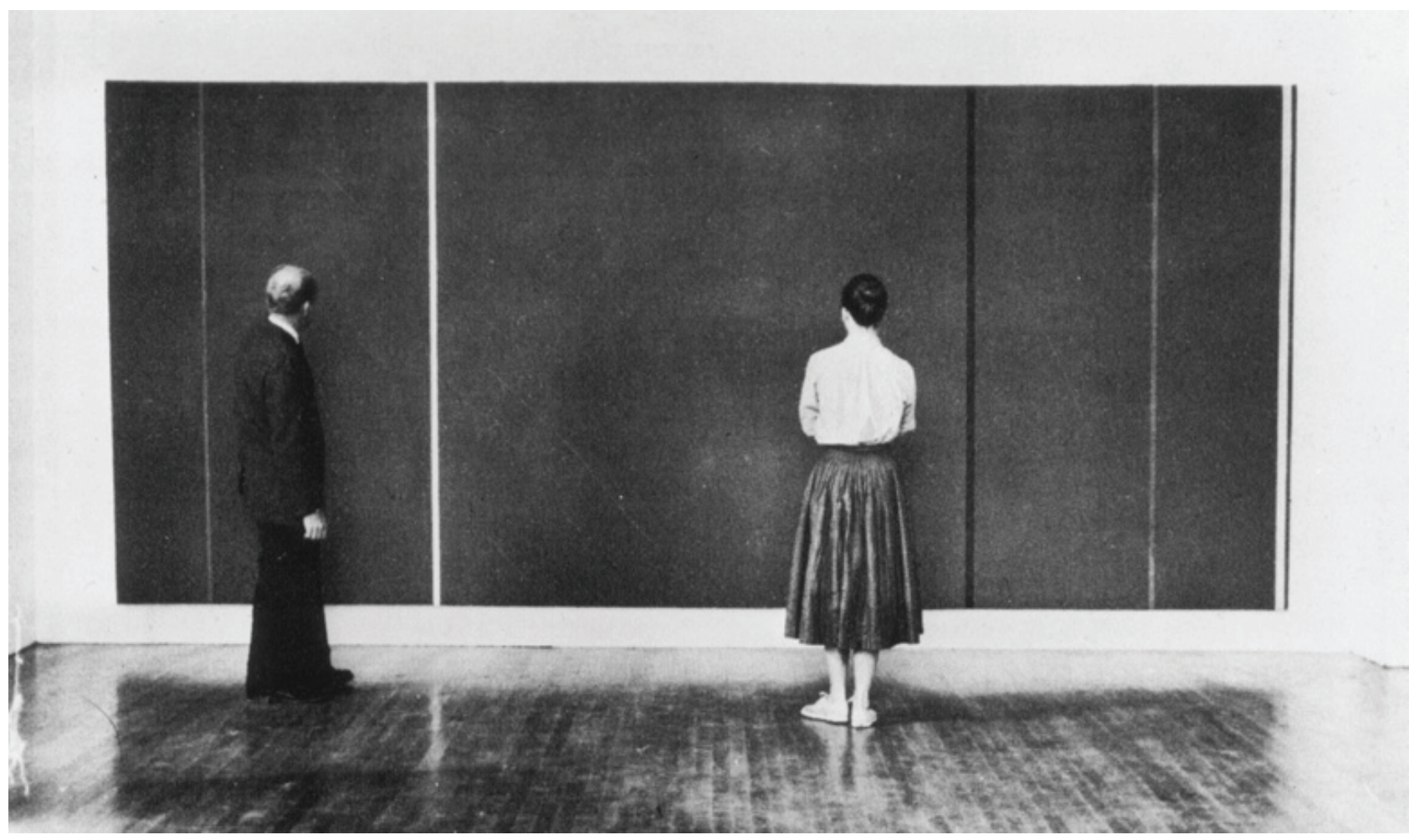

Abb. 4b. Betrachter vor Vir Heroicus Sublimis in der Ausstellung im Bennington College, Cermont 1958. Abgedruckt in Thomas B. Hess (1969): Barnett Newman. New York: Walker and Company, S. 50.

derts Bauern in Thailand gezeigt wurden. Die neue ,Rahmung', das Aufscheinen der Reproduktion als Bild im Bild, stellt neue Fragen eines individuellen, eines differenten Zugangs.

Wenn sich diese Fragen so abstrakt anhören, liegt es auf der Hand, dass sich der ausbreitende Diskurs um das Reenactment als Konkretisierung der Mechanismen von Reproduktion, Aktivierung, und Dokumentation anbietet. ${ }^{33}$ Die Wiederaufführung einer Performance unter neuen Bedingungen, in einem neuen Kontext, aufgrund einer histori-

33 Vgl. Arns / Horn 2007; Dreschke et al. 2016; Engelke 2017; Kartsaki 2016. 


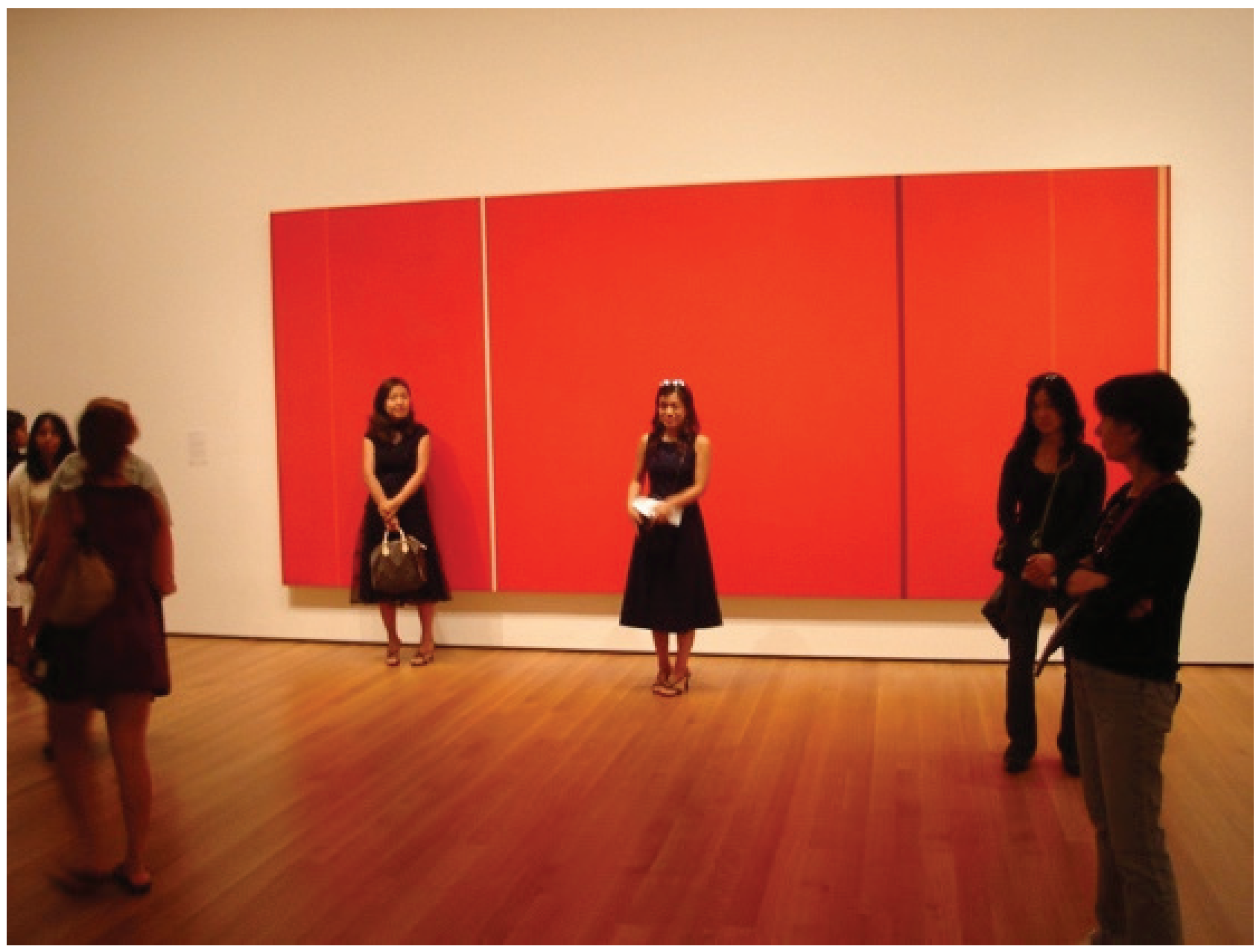

Abb. 4c. Besucherinnen lassen sich vor Barnett Newmans Vir Heroicus Sublimis im MoMA fotografieren.

Foto: Peter J. Schneemann

schen Dokumentation verdeutlicht die komplexen Verschränkungen zwischen Medientransfer, Bildfunktionen und Aktivierungsmomenten. Die Entscheidung, die ursprünglichen Performer_innen zu ersetzen und die Performance als Möglichkeit der Rekonstruktion oder der Fortführung zu wiederholen, zeigen Momente der Kopie an, die über die spezifischen Konstellationen der Performance hinausgehen. Die Kopie erweist sich als Manifestation eines jeweils spezifischen Moments der Lektüre und damit als Konstitution eines neuen Werkes abhängig von Publikum, Zeitkontext und Ortsbezogenheit. Wenn man sich Marina Abramović als bekanntestes Beispiel anschaut, wird man sich der Komplexität bewusst. Die ersten Videos ihrer Performances aus den 1970er Jahren wurden zunächst als Dokumentation diskutiert. ${ }^{34}$ Die ikonischen
Schwarz-Weiß-Screenshots der Zeit, also nicht nur jene von Abramović, wurden in der Folge bereits als eigenständige Werke wahrgenommen und in limitierten Editionen auf dem Kunstmarkt verkauft. Die meisten dieser Dokumentationsbilder waren gestellte Aufnahmen, häufig nachträglich, nach der Performance vor Publikum entstanden. Abramovićs Retrospektive The Artist is Present zeigte $2011 \mathrm{im}$ Museum of Modern Art Reenactments mit jungen Performerinnen und Performern. ${ }^{35}$ Dabei handelte es sich jedoch weniger um eine Wiederaufführung nach dem alten Script, als vielmehr um ein Aufgreifen der historischen Dokumentationsästhetik. Diese prägt das heutige Reenactment stärker als das Konzept einer Wiederaufführung. Marcel Bleuler hat in seiner Forschung zur Dokumentation von Performances überzeugend gezeigt, wie die präzise 


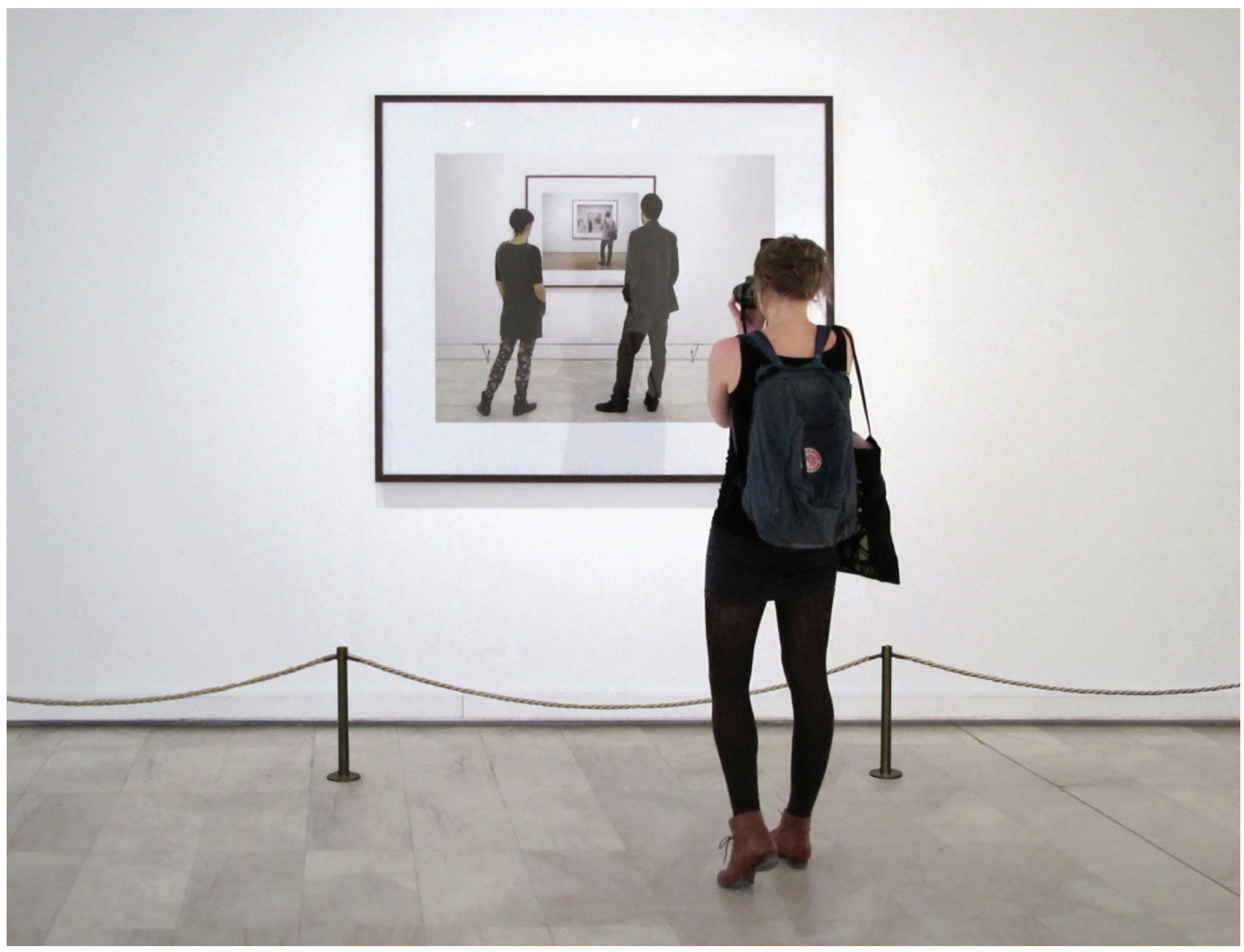

Abb. 5. Georgia Kotretsos, Being-seen-by-another is the truth of seeing the other, Macedonian Museum of Contemporary Art, Thessaloniki, Griechenland, 2011. Archival inkjet print. 75 x 87 cm, 2011, Courtesy of the artist.

zu benennende stilistische Eigenheit einer Dokumentation, mit Kameraführung und Ausschnitt, den Ausgangspunkt bildet für eine große, kollektive Aktivität auf YouTube oder verwandten Plattformen. ${ }^{36}$ Es handelt sich um die unzähligen individuellen Beiträge zum Weiterleben, zur Interpretation der kanonischen Performances.

Das Phänomen der Verschränkung von Performance und Handlung, Bild und Belebung und wiederholter Bildwerdung kennt weitere Wendungen: Wirft man einen Blick auf die Illustrationen im Katalog, Bilder von den Reenactments, wiederum in schwarz-weiß Ästhetik, findet sich die Bezeichnung für eine eigenartige Zwittergattung: Wir identifizieren nicht nur die Künstlerin in der Rolle einer Besucherin, sondern weitere Kuratorinnen und Kuratoren, die fiktiv und spie- lerisch als Statistinnen und Statisten die Rolle des Publikums übernehmen. Das Label benennt das Phänomen als,conceptual photographs': Es handelt sich nicht mehr um eine Dokumentation von einem Ereignis, sondern um eine Vorlage für das mögliche Rollenverhalten des neuen Publikums. Die Kopie dient damit als Matrix für ein sogenanntes Preenactment, wie es aktivistische Künstlerinnen, etwa Yael Bartana entwickelt haben. Die wiederholte Geste als Rekonstruktion greift nun als Präfiguration einer Differenz in die Zukunft. ${ }^{37}$ Die Kopie als Medium emanzipierter Rezeption mündet in komplexe Prozesse der Übersetzung, Abweichung und Auflösung.

Olaf Nicolai ließ in der Kunsthalle Wien 2018 auf einen eingezogenen Bühnenboden politische Pressebilder aus seinem Archiv von Pflasterma- 
lern und Auftragskünstlern in Kreide großformatig kopieren. Das Publikum war, ebenso wie diverse Performancekünstler_innen, dazu aufgefordert, diese Nachbildungen zu begehen, mit und auf innen zu agieren. Dieser performative Umgang hinterließ auf den Kopien Spuren - sie wurden sukzessive abgetragen. Ein Feedback-Loop trug Kopie und Prozess wiederum in die sozialen Medien und thematisierte damit die politische Dimension der in diesem Aufsatz diskutierten emanzipatorischen Potenzen der Kopie. ${ }^{38}$

\section{Literaturverzeichnis}

Armstrong, Elizabeth (Hg.) (2012): More Real? Art in the Age of Truthiness. Ausstellungskatalog, SITE Santa $\mathrm{Fe}, 08.07 .2012-06.01 .2013$. Minneapolis Institute of Arts Minneapolis, 03.03.-09.06.2013. München: Prestel.

Arns, Inke / Horn, Gabriele (Hgg.) (2007). History Will Repeat Itself. Strategien des Reenactment in der zeitgenössischen (Medien-)Kunst und Performance. Ausstellungskatalog, Dortmund, Phoenix Halle 09.06.23.09.2007. Berlin, KW Institute for Contemporary Art, 18.11.2007-13.01.2008. Frankfurt a. M.: Revolver.

Auslander, Phillip (2006): The Performativity of Performance Documentation. In: PAJ. A Journal of Performance and Art, 28/3, S. 1-10.

Bätschmann, Oskar (1984): Einführung in die kunstgeschichtliche Hermeneutik. Die Auslegung von Bildern. Darmstadt: Wissenschaftliche Buchgesellschaft.

Beyoncé / Jay-Z (2018): Apeshit. https://www.youtube. com/watch?v=kbMqWXnpXcA (29.05.2019).

Biesenbach, Klaus Peter (Hg.) (2010): Marina Abramović. The Artist is Present. Ausstellungskatalog, New York, Museum of Modern Art, 14.03-31.05.2010. London: Thames \& Hudson.

Bleuler, Marcel (2014): Deutungsvorschrift? Die filmische Vermittlung bei Marina Abramović und Pierre Huyghe. In: Ehninger, Eva / Nieslony, Magdalena (Hgg.): Theorie ${ }^{2}$. Potenziale und Potenzierungen künstlerischer Theorie. Bern / Frankfurt a. M.: Lang, S. 242-263.

Blunck, Lars (2011): Wann ist ein Original? In: Nida-Rümelin, Julian / Steinbrenner, Jakob (Hgg.): Original und Fälschung. Ostfildern: Hatje Cantz, S. 9-29.

Boime, Albert (1964): Le Musée des copies. In: Gazette des Beaux-Arts, 6/64, S. 237-247.

Castets, Simon / Coupland, Douglas / DIS (2014): \# artselfie. Paris: Jean Boîte.

Celant, Germano (Hg.) (2013): When Attitudes Become Form: Bern 1969/Venice 2013, Fondazione Prada, Ca' Corner della Regina, Venice 01.06.-03.11.2013. Mailand: Progetto Prada Arte.
Celant, Germano (2015): Re-Curating Attitudes. Bern 1969/Venice 2013. In: Smith, Terry (Hg.): Talking Contemporary Curating. New York: Independent Curators International, S. 248-277.

Conte, Kari (2018): Revivifying the Past. Exhibitions and Institutions. In: Schneemann, Peter J. (Hg.): Localizing the Contemporary. The Kunsthalle Bern as a Model. Zürich: JRP Ringier, S. 259-274.

Daur, Uta (Hg.) (2013): Authentizität und Wiederholung. Künstlerische und kulturelle Manifestationen eines Paradoxes. Bielefeld: transcript.

Dziewior, Yilmaz (2016): Blick durch die offene Fluchttür. In: Ders. (Hg.): Danh Võ. Ydob eht ni mraw si ti. Ausstellungskatalog, Köln, Museum Ludwig, 01.08.25.10.2015. Köln: Walther König, S. 13-24.

Dreschke, Anja / Huynh, Ilham / Knipp, Raphaela et al. (Hgg.) (2016): Reenactments. Medienpraktiken zwischen Wiederholung und kreativer Aneignung. Bielefeld: transcript.

Engelke, Heike (2017): Geschichte wiederholen. Strategien des Reenactment in der Gegenwartskunst. Omer Fast, Andrea Geyer und Rod Dickinson. Bielefeld: transcript.

Erben, Dietrich (2005): Der steinerne Gast. Die Begegnung mit Statuen als Vorgeschichte der Betrachtung. Weimar: VDG, S. 72-87.

Fehrmann, Gisela / Linz, Erika / Schumacher, Eckhard et al. (Hgg.) (2004): Originalkopie. Praktiken des Sekundären. Köln: DuMont.

Gelshorn, Julia (2012): Aneignung und Wiederholung. Bilddiskurse im Werk von Gerhard Richter und Sigmar Polke. München: Fink.

Grasskamp, Walter (2014): André Malraux und das imaginäre Museum. Die Weltkunst im Salon. München: Beck.

Gumbrecht, Hans Ulrich (2004a): Production of Presence: What Meaning Cannot Convey. Stanford, CA: Stanford University Press.

Gumbrecht, Hans Ulrich (2004b): Diesseits der Hermeneutik. Die Produktion von Präsenz. Frankfurt a. M.: Suhrkamp.

Han, Byung-Chul (2011): Shanzai. Dekonstruktion auf Chinesisch. Berlin: Merve.

Henningsen, Lena / Hofmann, Martin (Hgg.) (2012): Tradition? Variation? Plagiat? Motive und ihre Adaption in China. Wiesbaden: Harrassowitz.

Kartsaki, Eirini (Hg.) (2016): On Repetition. Writing, Performance \& Art. Chicago: University of Chicago Press.

Kernbauer, Eva (Hg.) (2015): Kunstgeschichtlichkeit. Historizität und Anachronie in der Gegenwartskunst. Paderborn: Fink.

Kihm, Christoph (2015): L'exposition et son spectateur. In: ArtPress2. Les expositions à l'ère de leur reproductibilité, 36 , S. 81-87. https://www.artpress. com/2015/02/10/sommaire-du-artpress-n36-les-expositions-a-lere-de-leur-reproductibilite (09.07.2019).

Kohle, Hubertus (2018): Museen digital. Eine Gedächtnisinstitution sucht den Anschluss an die Zukunft. Heidelberg: Heidelberg University Publishing.

38 Vgl. Nicolai 2018. 
Lambert, Susan (1987): The Image Multiplied. Five Centuries of Printed Reproductions of Paintings and Drawings. New York: Abaris Books.

Leader, Darian (Hg.) (2010): The Clock. Christian Marclay. Ausstellungskatalog, London, White Cube, 15.10.13.11.2010. London: White Cube.

Marchart, Oliver (2015): The Art of Pre-enactment. In: Malzacher, Florian (Hg.): Not Just a Mirror. Looking for the Political Theatre Today. Berlin: Alexander, S. 146-150.

Malraux, André (1949): Psychologie de l'Art: Le Musée imaginaire. Paris: Skira.

Malraux, André (1949 [1947]): Psychologie der Kunst. Das imaginäre Museum. Übersetzung von Jan Lauts. Baden-Baden: Klein.

Mensger, Ariane / Staatliche Kunsthalle Karlsruhe (Hgg.) (2012): Déjà-vu? Die Kunst der Wiederholung von Dürer bis YouTube. Bielefeld: Kerber.

Mersch, Dieter (2006): Hans Ulrich Gumbrecht. Diesseits der Hermeneutik. Die Produktion von Präsenz. In: Arbitrium, 23/3, S. 253-256.

Mundy, Jennifer (2013): Lost Art: Missing Artworks of the Twentieth Century. London: Tate Publishing.

Nicolai, Olaf (2018): There Is No Place Before Arrival. Ausstellungsbooklet, Kunsthalle Wien. Wien: Kunsthalle.

Roesler-Friedenthal, Antoinette / Nathan, Johannes (2003): The Enduring Instant. Time and the Spectator in the Visual Arts. Der bleibende Augenblick. Betrachterzeit in den Bildkünsten. Berlin: Gebr. Mann.

Rasdjarmrearnsook, Araya (2008): Dow Song Duang The Two Planets Series, Video, $17 \mathrm{~min}$.

Römer, Stefan (2001): Künstlerische Strategien des Fake. Kritik von Original und Fälschung. Köln: DuMont.

Santone, Jessica (2008): Marina Abramović's Seven Easy Pieces. Critical Documentation Strategies for Preserving Art's History. In: Leonardo, 41/2, S. 147-152.

Schneemann, Peter J. (2014): The Copy as a Reflection of Modes of Perception. Contemporary Artistic Practices and the Construction of Anagrammatic Images. In: Osano, Shigetoshi (Hg.): Between East and West. Reproductions in Art (Proceedings of the CIHA Colloquium in Naruto, Japan, 15.-18.1.2013). Krakau: IRSA, S. 95-107.

Schneemann, Peter J. (2019): The Revival of Ekphrasis. Rumours, Anecdotes and Descriptions as Strategies in Contemporary Art and Curating. In: The Silence of Images. Theories and Processes of Artistic Invention [MAXXI - Museo nazionale delle arti del XXI secolo. Rome. 11.-12.12.2015].
Schwartz, Hillel (1996): The Culture of the Copy. Striking Likenesses, Unreasonable Facsimiles. New York: Zone Books.

Schwartz, Hillel (2000): Déjà vu. Die Welt im Zeitalter ihrer tatsächlichen Reproduzierbarkeit. Berlin: Aufbau.

Schweizer, Nicole (Hg.) (2017): Yael Bartana. Zürich: JRP Ringier.

Sheldon, Julie (2017): Picturing the Installation Shot. In: Meecham, Pam (Hg.): A Companion to Modern Art. Hoboken: Wiley, S. 127-143.

Struth, Thomas / Belting, Hans / Grasskamp, Walter (1993): Thomas Struth. Museum Photographs. Ausstellungskatalog, Hamburg, Kunsthalle, 11.11.1993-16.01.1994. München: Schirmer / Mosel.

Tietenberg, Annette (Hg.) (2015): Die Ausstellungskopie. Mediales Konstrukt, Materielle Rekonstruktion, Historische Dekonstruktion. Köln / Weimar / Wien: Böhlau.

Ullrich, Wolfgang (2015): Raffinierte Kunst. Übung vor Reproduktionen. Berlin: Wagenbach.

von Bismarck, Beatrice (2014): Out of Sync, or Curatorial Heterochronicity. ,Anti-Illusion: Procedures/Materials' (1969). In: Bismarck, Beatrice von / Frank, Rike / Meyer-Kramer, Benjamin et al. (Hgg.): Timing. On the Temporal Dimension of Exhibiting. Berlin: Sternberg Press, S. 301-318.

von Gehlen, Dirk (2012 [2011]): Mashup. Lob der Kopie. Berlin: Suhrkamp.

Weibel, Peter (Hg.) (1999): Kunst ohne Unikat. Multiple und Sampling als Medium. Techno-Transformationen der Kunst. Köln: König.

Zentrum für Kunst und Medientechnologie (2011): The Global Contemporary. Kunstwelten nach 1989. Ausstellungskatalog, Karlsruhe und Akademie der Künste Berlin: ZKM.

Zentrum für Kunst und Medientechnologie (2013): Nothing to Declare? World Maps of Art since 89. https://www.adk.de/de/projekte/2013/Nothing_to_ declare/Programm.htm (19.07.2019). Karlsruhe und Akademie der Künste Berlin: ZKM. 